(6) OPEN ACCESS

\title{
Dual-target, real-time PCR for the diagnosis of intraocular Toxoplasma gondii infections
}

\author{
Carlos A Gomez, ${ }^{1,2}$ Malaya K Sahoo, ${ }^{3}$ Ghazala Yasmeen Kahn, ${ }^{4}$ Lina Zhong, ${ }^{5}$ \\ José G Montoya, ${ }^{1,2}$ Benjamin A Pinsky, 1,3,4 Thuy Doan ${ }^{5,6}$
}

${ }^{1}$ Division of Infectious Diseases and Geographic Medicine, Department of Medicine, Stanford University School of Medicine, Stanford, California, USA

${ }^{2}$ Palo Alto Medical Foundation Toxoplasma Serology

Laboratory, National Reference Center for the Study and

Diagnosis of Toxoplasmosis, Palo Alto, California, USA

${ }^{3}$ Department of Pathology, Stanford University School of Medicine, Stanford, California, USA

${ }^{4}$ Clinical Virology Laboratory, Stanford Health Care and Stanford Children's Health Stanford, California, USA ${ }^{5}$ F.I. Proctor Foundation, University of California San Francisco, San Francisco, California, USA

${ }^{6}$ Department of Ophthalmology, University of California, San Francisco, California, USA

\section{Correspondence to} Dr Benjamin A Pinsky, Department of Pathology, Stanford University School of Medicine, Stanford, CA 94305, USA; bpinsky@stanford.edu

Received 13 August 2018 Revised 25 October 2018 Accepted 14 November 2018 Published Online First 12 January 2019

\section{ABSTRACT}

Background Toxoplasma gondii is the most common infectious cause of posterior uveitis worldwide. Two multicopy targets (B1 and Rep529) are commonly used in T. gondii PCR assays, but studies evaluating these targets in ocular fluid samples are limited. Herein, we determine the analytical characteristics of a singlereaction, internally controlled, dual-target, real-time $T$. gondii PCR and evaluate the clinical performance of this assay in intraocular fluid samples obtained at a reference ophthalmologic centre in the USA.

Methods Lower limits of detection for the B1 and Rep529 components of the dual-target assay were determined using serial dilutions of cultured T. gondii strain Z185. The dual-target assay was then used to test 148 archived intraocular samples (132 vitreous, 16 aqueous humour) collected at the Francis I. Proctor Foundation between January 2010 and December 2015 for testing by a nested, conventional PCR targeting the B1 gene.

Results The $95 \%$ lower limits of detection for the dualtarget assay was determined to be 1.05 tachyzoites/ $\mathrm{mL}$ for B1 and 0.83 tachyzoites/mL for Rep529. Using archived clinical intraocular specimens, the dualtarget assay demonstrated $97.2 \%$ positive per cent agreement ( $n=35 / 36 ; 95 \% \mathrm{Cl} 83.7 \%$ to $99.9 \%$ ) and 99.1\% negative per cent agreement $(n=111 / 112$; $95 \%$ Cl $94.4 \%$ to $100 \%$ ) compared with the nested, conventional B1 PCR.

Conclusion This single-reaction, internally controlled, dual-target (B1, Rep529) real-time PCR for the detection of $T$. gondii DNA in intraocular specimens demonstrated excellent agreement with nested, conventional, B1 PCR. The dual-target design may ensure $T$. gondii detection when variation is present in one of two target regions.

\section{INTRODUCTION}

Toxoplasma gondii is the leading infectious cause of posterior uveitis worldwide and may result in significant morbidity and vision loss, particularly if there is a delay in diagnosis and treatment. ${ }^{12}$ Ocular toxoplasmosis may occur in the setting of congenital or otherwise distant $T$. gondii infection, but may also present following primary infection. ${ }^{34}$ Ordinarily, fundus examination alone is sufficient to establish the diagnosis of ocular toxoplasmosis. When clinical presentation is atypical, particularly in immunocompromised patients, or when the fundus is not well visualised due to dense vitritis or other opacities, or in cases refractive to anti-toxoplasma therapy, laboratory testing of intraocular fluid samples is indicated to confirm the presence of T. gondii DNA and rule out other infectious causes.

Molecular testing for T. gondii DNA relies on the detection of two conserved multicopy sequences present in the $T$. gondii genome; the B1 gene (30-35 copies/genome) and the Rep529 repetitive elements (200-300 copies/genome). ${ }^{5}$ While anterior chamber paracentesis and vitreous tap can be routinely done in the clinic to obtain intraocular fluid for testing, they are not without the potential for procedure-associated complications (eg, corneal endothelium or lens injury, intraocular haemorrhage, retinal detachment and endophthalmitis). Coupled with the low sample volumes that can be safely obtained from the patient, it is highly desirable to have the selection of an assay target with the best diagnostic performance. Due to a higher genomic copy number, analytical and clinical sensitivity for assays targeting Rep529 appear to be superior to B1 assays; however, few ocular samples have been included in these studies. ${ }^{5-7}$ To date, direct comparisons assessing the diagnostic performance of PCR assays targeting B1 and Rep529 targets in ocular samples from US patients, where different T. gondii strains are prevalent, ${ }^{8}$ have not been performed. Herein, we describe the analytical characteristics of a single-reaction, internally controlled, B1-Rep529 dual-target, real-time PCR assay and evaluate the clinical performance of this assay on intraocular fluid samples collected at the Francis I. Proctor Foundation, a tertiary centre for ophthalmologic care in the USA.

\section{METHODS \\ Study design}

One hundred and forty-eight archived intraocular samples (132 vitreous fluid, 16 aqueous humour) previously tested for $T$. gondii DNA at the Francis I. Proctor Foundation were selected for evaluation. The samples were archived between January 2010 and December 2015, boiled, and then stored at $-80^{\circ} \mathrm{C}$. The samples were deidentified using standard institutional procedures and randomised. All laboratory personnel were masked to the reference results until all analyses were completed.

\section{Lower limit of detection}

Partially purified T. gondii strain Z185 culture fluid from propagation in MRC-5 fibroblast cells was purchased from Zeptometrix (Buffalo, New York, USA). The tachyzoites were quantitated using a Petroff-Hausser Counting Chamber, and the stock concentration determined to be $6.6 \times 10^{6}$ 
A Dual-Target $T$. gondii Multiplex, Real-time PCR, hydrolysis probes

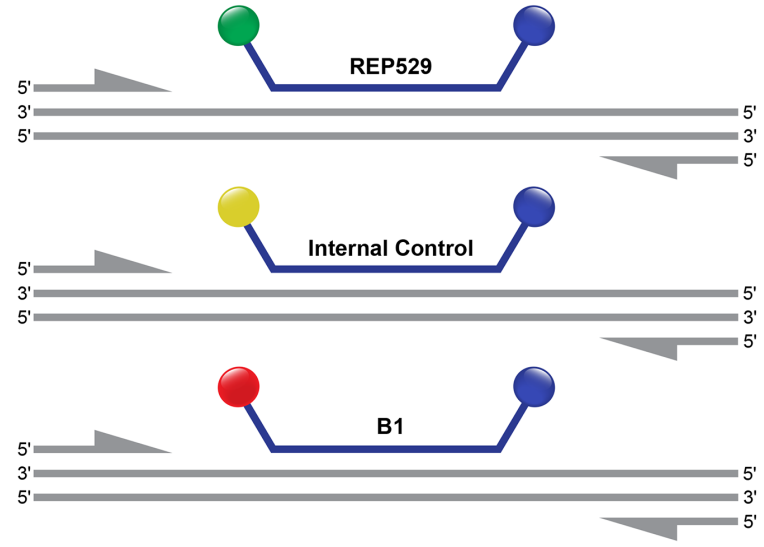

B

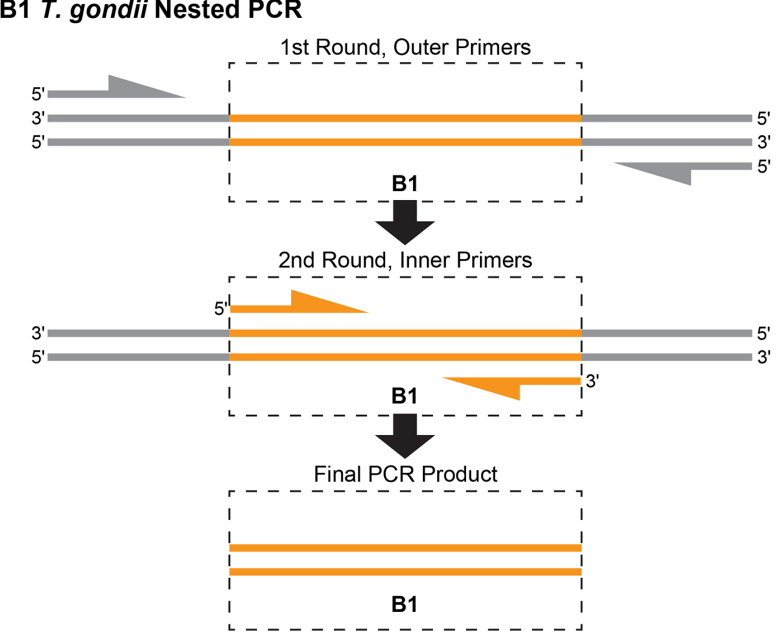

Figure 1 Comparison of dual-target Toxoplasma gondii multiplex, real-time PCR and B1 T. gondii nested PCR. (A) The dual-target assay uses fluorescently labelled hydrolysis probes targeting B1 (crimson) and Rep529 (green) as well as an internal control (yellow). This design allows amplification and fluorescence detection of both B1 and Rep529 in a single, real-time PCR reaction. (B) The nested B1 PCR assay relies on sequential conventional $P C R$ reactions using outer primers in the first reaction and inner primers in the second reaction. The final PCR product is detected by agarose gel electrophoresis and ethidium bromide staining.

tachyzoites $/ \mathrm{mL}$. The 95\% lower limit of detection (LLOD) was calculated by probit analysis using the Generalized Linear Models (glm) function from stats package (V.3.3.1) in R. ${ }^{9}$

\section{T. gondii single-reaction, internally-controlled, dual-target, real-time PCR assay}

Total nucleic acids were extracted from $50 \mu \mathrm{L}$ culture supernatant dilutions or up to $50 \mu \mathrm{L}$ of each clinical specimen on the BioRobot EZ1 (Qiagen, Germantown, Maryland, USA) using the EZ1 virus mini kit 2.0 according to the manufacturer's recommendations. The sample volume was made up to $200 \mu \mathrm{L}$ with nuclease-free water and eluted in $60 \mu \mathrm{L}$ buffer AVE containing cRNA. Internal control (IC) DNA template was supplied at this step which is spiked in and extracted by the instrument.

ThisPCRassaytargetsbothB1 andRep529(figure 1A) usingprimers and probes described previously. ${ }^{10}$ Primers (Rep529_F: 5'-AGAG ACACCGGAATGCGATCT-3'; Rep529_R: 5'-TTCGTCCAAGCCTCCGACT-3'; B1_F: 5'-GAAAGCCĀTGAGGCACTCCA-3'; B1_R: 5'-TTCACCCGGACCGTTTAGC-3') were purchased from
Integrated DNA Technologies (San Diego, California, USA) and hydrolysis probes (Rep529 probe: 5'-FAM-TCGTGGTGATGG CGGAGAGAATTGA-BHQ1-3'; B1 Probe: 5'-Quasar 705-CGGG CGAGTAGCACCTGAGGAGATACA-BHQ2-3') were purchased from Biosearch Technologies (Petaluma, California, USA). Realtime PCR was performed using the Quantifast Pathogen + IC kit on the Rotor-Gene Q instrument (both from Qiagen, Germantown, Maryland, USA). Primers and probes were added in a single reaction at final concentrations of $400 \mathrm{nM}$ and $200 \mathrm{nM}$, respectively. Ten $\mu \mathrm{L}$ of eluate was added in a final reaction volume of $25 \mu \mathrm{L}$. The Quantifast Pathogen + IC master mix also contains a primer/ probe set targeting the IC DNA. Cycling conditions are as follows: hold at $95^{\circ} \mathrm{C}$ for $5 \mathrm{~min}$, then 45 cycles of $95^{\circ} \mathrm{C}$ for $15 \mathrm{~s}$ and $60^{\circ} \mathrm{C}$ for $30 \mathrm{~s}$. Detection was performed in the green (Rep529), crimson (B1) and yellow (IC) channels; the threshold was set at 0.05 for all three channels. Any amplification curve crossing this threshold in green or crimson channels was considered positive for $T$. gondii. Samples were considered to have failed extraction or contain inhibitory substances if the IC did not amplify in samples negative for T. gondii.

\section{Reference testing}

Nested, conventional PCR targeting the B1 gene (figure 1B) was performed at the Francis I. Proctor Foundation CLIA-accredited microbiology laboratory as part of routine clinical testing. Briefly, intraocular fluid samples were boiled for $10 \mathrm{~min}$ and $4 \mu \mathrm{L}$ of the boiled aqueous or vitreous fluid was subjected to 2 rounds of PCR with the following primers targeting the B1 gene: 5F_external: GGA ACT GCA TCC GTT CAT GAG; 5R_external: TCT TTA AAG CGT TCG TGG TC; 5F_internal: TGC ATA GGT TGC AGT CAC TG; 5R_internal: GGC GAC CAA TCT GCG AAT ACA CC. Both PCR assays used $5 \mu \mathrm{L}$ of 10X PCR buffer (Sigma-Aldrich, St. Louis, Missouri, USA), 1.5 $m \mathrm{M}$ of $\mathrm{MgCl}_{2}, 0.1 \mathrm{mM}$ dNTP mix, $10 \mathrm{pmol}$ of each primer and $0.05 \mathrm{U}$ of REDTaq DNA polymerase (Sigma-Aldrich) in a total reaction volume of $50 \mu \mathrm{L}$. Amplified products were evaluated on 4\% e-Gels (Thermo Fisher Scientific, Waltham, Massachusetts, USA). This assay has a LLOD of 5 tachyzoites $/ \mathrm{mL}$ (unpublished, F. I. Proctor Foundation).

Samples in which the nested PCR and dual-target, real-time PCR results were discrepant, were tested by B1 real-time PCR at the Palo Alto Medical Foundation Toxoplasmosis Serology Laboratory (PAMF-TSL), the US National Toxoplasmosis Reference Laboratory.

\section{RESULTS}

The 95\% LLOD of dual-target assay was determined to be 1.05 tachyzoites/mL for B1 and 0.83 tachyzoites/mL for Rep529. A set of 148 intraocular specimens, 132 vitreous and 16 aqueous samples, collected from patients with suspected intraocular $T$. gondii infection were then tested by the dual-target assay. Appropriate amplification of the IC in all samples revealed adequate extraction and the absence of inhibitors. Compared with the reference nested B1 PCR performed at the F.I. Proctor Foundation, the dual-target assay demonstrated $97.2 \%$ positive agreement $(\mathrm{n}=35 / 36 ; 95 \%$ CI $83.7 \%$ to $99.9 \%)$ and $99.1 \%$ negative agreement ( $\mathrm{n}=111 / 112 ; 95 \%$ CI $94.4 \%$ to $100 \%)$ (table 1$)$. There were 35 samples with detectable T. gondii DNA in both assays, and in all of these samples, both the B1 and Rep529 targets were detected in the dual-target assay. The cycle threshold value was on average 0.75 cycles later in the crimson channel (B1) compared with the green channel (Rep529) $(\mathrm{p}=0.0019,95 \% \mathrm{CI}$ 0.30 to 1.20 ; paired t-test). 
Table 1 Comparison of Toxoplasma gondii DNA detection in the B1-Rep529 dual-target, real-time PCR assay and the nested, conventional B1 PCR performed at the Francis I. Proctor Foundation

\begin{tabular}{lllcc}
\hline & & \multicolumn{2}{l}{ Nested B1 PCR } & \\
\cline { 3 - 4 } & & Positive & Negative & Total \\
\hline B1-Rep529 & Positive & 35 & 1 & 36 \\
real-time PCR & Negative & 1 & 111 & 112 \\
& Total & 36 & 112 & 148 \\
\hline
\end{tabular}

There were two discrepant samples; one dual-target negative, nested B1 positive and the other dual-target positive (Rep529 only; crossing threshold $(\mathrm{Ct})$ value $=39.14)$, nested B1 negative. Reference testing at the PAMF-TSL demonstrated that both specimens were negative for T. gondii DNA using B1 real-time PCR.

\section{DISCUSSION}

We present here the analytical and clinical evaluation of a single-reaction, internally controlled, B1-Rep529 dual-target T. gondii real-time PCR assay for the diagnosis of ocular toxoplasmosis. As expected, the Rep529 component of this assay was more analytically sensitive than the B1 component, as has been demonstrated in several manuscripts comparing B1 and Rep529 single-target assays. ${ }^{11-14}$ We also observed excellent overall agreement of the dual-target assay compared with conventional, nested B1 PCR when used to test intraocular specimens from patients with suspected ocular toxoplasmosis.

This study comparatively evaluates the diagnostic performance of the dual-target $T$. gondii-PCR assay on intraocular samples from US patients (table 2). In Europe, assays targeting Rep529 have largely replaced assays targeting B1 due to higher genome copy numbers (200-300 copies/genome vs 30-35 copies/ genome) and overall higher clinical sensitivity in numerous specimen types, including amniotic fluid, placental tissue, cord blood, cerebrospinal fluid and peripheral blood. However, few studies have directly compared B1 and Rep529 PCR assays on intraocular specimens. A literature review performed in PubMed of studies published in the English language identified only four such studies, three conducted in France and one in Brazil (table 2). ${ }^{10131516}$ Though these studies used various reference methods and included a wide range of sample sizes, Rep529 DNA was generally detected in more cases than B1 DNA. Our study included one B1 negative/Rep529 positive case (Ct value $=39.14)$; a false negative B1 case was also reported by Belaz et al in an intraocular specimen with a late Rep529 Ct value. ${ }^{13}$ These findings suggest that low parasite loads, which can occur in the setting of prior anti-toxoplasma therapy, may account for these B1 negative/Rep529 positive cases. Consistent with this hypothesis, several studies of congenital toxoplasmosis in France, where anti-toxoplasma therapy is offered throughout pregnancy, have demonstrated lower diagnostic sensitivity of B1 PCR assays in amniotic fluid samples. ${ }^{17} 18$ Consequently, exposure to anti-toxoplasma therapy in ocular toxoplasmosis may have the same deleterious effect on the diagnostic yield of intraocular T. gondii PCR if tested using a B1-only assay.

In contrast to the previous studies investigating T. gondii PCR targets in intraocular fluids, the assay described herein combines B1, Rep529 and an IC in a single reaction. Nevertheless, our data, as well as the studies presented in table 2, support the implementation of a diagnostic assay that targets only Rep529. However, this single-reaction, dual-target approach is frequently applied in molecular diagnostics for viral infections, such as HIV-1 and HBV, to reduce the chance of false-negative results due to mutations that disrupt primer/probe binding. The utility of an assay that includes both B1 and Rep529 targets in a single tube, is supported by a report from East Africa in which 5\% of clinical samples demonstrated Rep529 amplification failures. ${ }^{19}$ Similarly, a study of amniotic fluid specimens collected in Brazil demonstrated a lower proportion of positive results detected by the Rep529 target (36.5\%) compared with the B1 target $(87.3 \%)$ with only $24 \%$ of samples being positive for both targets. ${ }^{20}$ In our US cohort, however, we did not identify any dual-target-assay-positive intraocular samples in which only the B1 target was detected. Nonetheless, as ocular toxoplasmosis frequently emerges from congenital or postnatally acquired T. gondii infections, diagnosis of B1 positive/Rep529 negative ocular toxoplasmosis may be particularly important in patients born outside of the USA and in returning travellers. In addition, some T. gondii strains may contain fewer copies of Rep529 than previously estimated, ${ }^{21}$ further supporting the inclusion of both targets to optimise $T$. gondii DNA detection.

Table 2 Studies evaluating real-time PCR assays for detection of Toxoplasma gondii DNA in intraocular samples using the B1 gene and Rep529 targets

\begin{tabular}{|c|c|c|c|c|c|c|c|c|c|c|c|}
\hline \multirow[b]{2}{*}{ Author } & \multirow[b]{2}{*}{ Year } & \multirow[b]{2}{*}{ Country } & \multirow{2}{*}{$\begin{array}{l}\text { Single- } \\
\text { reaction }\end{array}$} & \multirow{2}{*}{$\begin{array}{l}\text { Internally } \\
\text { controlled }\end{array}$} & \multirow{2}{*}{$\begin{array}{l}\text { Sample } \\
\text { size }\end{array}$} & \multirow[b]{2}{*}{ Type of fluid } & \multicolumn{2}{|c|}{ Sensitivity $\mathrm{n} / \mathrm{N}(\%)$} & \multicolumn{2}{|l|}{$\begin{array}{l}\text { Specificity } \\
(\%)\end{array}$} & \multirow[t]{2}{*}{ Reference test } \\
\hline & & & & & & & B1 & Rep529 & B1 & Rep529 & \\
\hline Fekkar et a $\left.\right|^{10}$ & 2008 & France & No & Yes & 110 & AH:92; VF: 18 & $9 / 34(26.5 \%)$ & $13 / 34(38.2 \%)$ & $76 / 76(100 \%)$ & $76 / 76(100 \%)$ & $\begin{array}{l}\text { Ophthalmological } \\
\text { examination }\end{array}$ \\
\hline Belaz et $a l^{13}$ & 2015 & France & No & Yes & 6 & $\begin{array}{l}\text { AH:5; } \\
\text { VF:1 }\end{array}$ & $5 / 6(83.3 \%)$ & $6 / 6(100 \%)$ & NA & NA & Western blot \\
\hline Gomez & $C R$ & USA & Yes & Yes & 148 & AH:16;VF:132 & $35 * / 36(97.2 \%)$ & $35 * / 36(97.2 \%)$ & $112 / 112(100 \%)$ & $111 * / 112(99 \%)$ & $\begin{array}{l}\text { Conventional nested } \\
\text { B1 PCR }\end{array}$ \\
\hline
\end{tabular}

*There were two discrepant samples; one dual-target negative, nested B1 positive, and the other dual-target positive (Rep529 only; Ct value=39.14), nested B1 negative. Reference testing at the Palo Alto Medical Foundation Toxoplasmosis Serology Laboratory revealed that both specimens were negative for $T$. gondii DNA using B1 real-time PCR. $\mathrm{AH}$, aqueous humour; $\mathrm{CR}$, current report; NA, not available; VF, vitreous fluid. 
Limitations of this study include its retrospective design, and the absence of clinical, serological and epidemiological data, to correlate with molecular target detection. Factors such as the presence or length of anti-toxoplasma therapy, the timing between the onset of clinical symptoms and ocular fluid sampling, as well as severity and rates of relapse, were not investigated, though these variables may explain the overall similar performance of the two targets. In addition, immunodiagnostic methods, such as the Goldmann-Witmer coefficient and Western blotting, which may be complementary to nucleic acid amplification testing, were not performed. Finally, typing and genomic characterisation of the $T$. gondii strains causing ocular toxoplasmosis in the USA were beyond the scope of this study, particularly in the context of type-specific associations with severe disease and higher rates of recurrences. ${ }^{22}$ Comparison of these strains with T. gondii genomes from other parts of the world may yield a better understanding of the pathogenesis of ocular toxoplasmosis, as well as improved strategies for diagnosis and therapy.

In conclusion, we describe herein a single-reaction, internally controlled, B1-Rep529 dual-target T. gondii real-time PCR assay for the diagnosis of ocular toxoplasmosis. This single-reaction, dual-target design maximises inclusivity and sensitivity, without loss of specificity.

Acknowledgements The authors would like to thank the Francis I. Proctor Foundation staff as well as the staff of the Stanford Clinical Virology Laboratory for their diligent work and dedication to patient care.

Contributors BAP and TD conceived and planned the experiments and obtained IRB approvals. MKS, GYK and LZ contributed to sample preparation and carried out the experiments. CAG, MKS, JGM, BAP and TD contributed to the interpretation of the results. CAG took the lead in writing the manuscript. All authors provided critical feedback and helped shape the research, analysis and manuscript.

Funding This study was supported by the National Eye Institute of the National Institutes of Health (award no. K08EY026986 to TD) and the Research to Prevent Blindness Career Development Award (to TD).

Competing interests None declared.

Patient consent for publication Not required.

Ethics approval This study was approved by the Institutional Review Boards of the University of California San Francisco and Stanford University.

Provenance and peer review Not commissioned; externally peer reviewed.

Open access This is an open access article distributed in accordance with the Creative Commons Attribution Non Commercial (CC BY-NC 4.0) license, which permits others to distribute, remix, adapt, build upon this work non-commercially, and license their derivative works on different terms, provided the original work is properly cited, appropriate credit is given, any changes made indicated, and the use is non-commercial. See: http://creativecommons.org/licenses/by-nc/4.0

\section{REFERENCES}

1 Maenz M, Schlüter D, Liesenfeld 0 , et al. Ocular toxoplasmosis past, present and new aspects of an old disease. Prog Retin Eye Res 2014;39:77-106.
2 Jones JL, Holland GN. Annual burden of ocular toxoplasmosis in the US. Am J Trop Med Hyg 2010;82:464-5.

3 Jones JL, Bonetti V, Holland GN, et al. Ocular toxoplasmosis in the United States: recent and remote infections. Clin Infect Dis 2015;60:271-3.

4 Moshfeghi DM, Dodds EM, Couto CA, et al. Diagnostic approaches to severe, atypical toxoplasmosis mimicking acute retinal necrosis. Ophthalmology 2004; 111:716-25

5 Doan T, Pinsky BA. Current and future molecular diagnostics for ocular infectious diseases. Curr Opin Ophthalmol 2016;27:561-7.

6 Homan WL, Vercammen M, De Braekeleer J, et al. Identification of a 200- to 300-fold repetitive 529 bp DNA fragment in Toxoplasma gondii, and its use for diagnostic and quantitative PCR. Int J Parasitol 2000;30:69-75.

7 Robert-Gangneux F, Dardé ML. Epidemiology of and diagnostic strategies for toxoplasmosis. Clin Microbiol Rev 2012;25:264-96.

8 Pomares C, Devillard S, Holmes TH. Genetic characterization of Toxoplasma gondii DNA samples isolated from humans Living in North America: an unexpected high prevalence of atypical Genotypes. J Infect Dis 2018;218:1783-91.

9 Venables WN, Ripley BD, Venables WN. Modern applied statistics with S. 4th edn. New York: Springer, 2002.

10 Fekkar A, Bodaghi B, Touafek F, et al. Comparison of immunoblotting, calculation of the Goldmann-Witmer coefficient, and real-time PCR using aqueous humor samples for diagnosis of ocular toxoplasmosis. J Clin Microbiol 2008;46:1965-7.

11 Delhaes L, Yera $\mathrm{H}, \mathrm{Ach} \mathrm{S}$, et al. Contribution of molecular diagnosis to congenital toxoplasmosis. Diagn Microbiol Infect Dis 2013;76:244-7.

12 Reischl U, Bretagne S, Krüger D, et al. Comparison of two DNA targets for the diagnosis of Toxoplasmosis by real-time PCR using fluorescence resonance energy transfer hybridization probes. BMC Infect Dis 2003;3:7.

13 Belaz S, Gangneux JP, Dupretz P, et al. A 10-year retrospective comparison of two target sequences, REP-529 and B1, for Toxoplasma gondii detection by quantitative PCR. J Clin Microbiol 2015:53:1294-300.

14 Kasper DC, Sadeghi K, Prusa AR, et al. Quantitative real-time polymerase chain reaction for the accurate detection of Toxoplasma gondii in amniotic fluid. Diagn Microbiol Infect Dis 2009;63:10-15.

15 Cassaing $\mathrm{S}$, Bessières $\mathrm{MH}$, Berry $\mathrm{A}$, et al. Comparison between two amplification sets for molecular diagnosis of toxoplasmosis by real-time PCR. J Clin Microbiol 2006:44:720-4.

16 Santos FF, Nascimento H, Muccioli C, et al. Detection of toxoplasma gondii DNA in peripheral blood and aqueous humor of patients with Toxoplasmic active focal necrotizing retinochoroiditis using real-time PCR. Arq Bras Oftalmol 2015:78:356-8.

17 Romand S, Chosson M, Franck J, et al. Usefulness of quantitative polymerase chain reaction in amniotic fluid as early prognostic marker of fetal infection with Toxoplasma gondii. Am J Obstet Gynecol 2004;190:797-802.

18 Peyron F, Mc Leod R, Ajzenberg D, et al. Congenital Toxoplasmosis in France and the United States: one parasite, two diverging approaches. PLoS Negl Trop Dis 2017; 11:e0005222.

19 Wahab T, Edvinsson B, Palm D, et al. Comparison of the AF146527 and B1 repeated elements, two real-time PCR targets used for detection of Toxoplasma gondii. J Clin Microbiol 2010;48:591-2.

20 Okay TS, Yamamoto L, Oliveira LC, et al. Significant performance variation among PCR systems in diagnosing congenital toxoplasmosis in São Paulo, Brazil: analysis of 467 amniotic fluid samples. Clinics 2009;64:171-6.

21 Costa JM, Bretagne S. Variation of B1 gene and AF146527 repeat element copy numbers according to Toxoplasma gondii strains assessed using real-time quantitative PCR. J Clin Microbiol 2012:50:1452-4.

22 Shobab L, Pleyer U, Johnsen J, et al. Toxoplasma serotype is associated with development of ocular toxoplasmosis. J Infect Dis 2013:208:1520-8. 